\title{
Formação de redes de governança para a inovação no setor público: estudo da Rede InovaGov e Comunidade de Simplificação
}

\author{
Cristina Camila Teles Saldanha ${ }^{1}$ \\ Ana Luiza Santos Terra ${ }^{1}$ \\ Marcus Vinicius Gonçalves da Cruz $^{2}$ \\ Carolina Gomes Rosado ${ }^{3}$ \\ José Clemente Maria Ferreira Santos ${ }^{1}$ \\ ${ }^{1}$ Universidade Federal de Minas Gerais (UFMG), Belo Horizonte - MG, Brasil \\ ${ }^{2}$ Fundação João Pinheiro (FJP), Belo Horizonte - MG, Brasil \\ ${ }^{3}$ Universidade Federal de Viçosa (UFV), Viçosa - MG, Brasil
}

O artigo busca compreender a formação de redes de governança voltadas para inovação governamental no Brasil a partir de um estudo de dois casos: a Rede InovaGov do Governo Federal e a Comunidade de Simplificação do Governo de Minas Gerais. Por meio de pesquisa de natureza qualitativa e descritiva, os dados foram analisados considerando duas dimensões de análise: a dos atores e a do processo. As variáveis qualitativas utilizadas para a dimensão dos atores foram: participantes, responsáveis e nível de engajamento; enquanto as do eixo processo foram: o tempo de existência das redes, o tipo de interação e o processo decisório de ambas. Concluiu-se que as diferenças entre as redes são relativas à maturidade, à amplitude da rede federal e à similaridade dos atores da rede estadual, mesmo que ambos sejam fenômenos recentes. Como proposta para futuras pesquisas, sugere-se estudo longitudinal que acompanhe as redes no seu processo de institucionalização.

Palavras-chave: inovação no setor público, redes de governança, redes de inovação 
Formación de redes de gobierno para la innovación en el sector público: estudio de la Rede InovaGov y la Comunidade de Simplificação

El artículo busca comprender la formación de redes de gobernanza dirigidas a la innovación gubernamental en Brasil a partir del estudio de dos casos: la Rede InovaGov del Gobierno Federal y la Comunidade de Simplificação del Gobierno de Minas Gerais. Por medio de una investigación de naturaleza cualitativa y descriptiva, los datos fueron analizados considerando dos dimensiones de análisis: la de los actores y la del proceso. Las variables cualitativas utilizadas para la dimensión de los actores fueron participantes, responsables y nivel de compromiso, mientras que las del eje proceso fueron el tiempo de existencia de las redes, el tipo de interacción y el proceso decisorio de ambas. Se concluyó que las diferencias entre ambas son relativas a la madurez, a la amplitud de la red de gobernanza federal y a la similitud de los actores de la red de gobernanza estatal, aunque ambas sean fenómenos recientes. Como propuesta para futuras investigaciones, se sugiere un estudio longitudinal que acompañe a las redes en su proceso de institucionalización.

Palabras clave: innovación en el sector público, redes de gobernanza, redes de innovación

Formation of governance networks for innovation in the public sector: study of the Rede InovaGov and Comunidade de Simplificação

The paper aims to understand the formation of governance networks geared towards governmental innovation in Brazil based on a study of two cases: the Rede InovaGov of the Federal Government and the Comunidade de Simplificação of the Minas Gerais government. Through qualitative and descriptive research, the data were analyzed considering two dimensions of analysis: the actors and the process. The qualitative variables used for the dimension of the actors were participants, responsible and level of engagement, while those of the process axis were the time of existence of the networks, the type of interaction and the decision making process of both. It was concluded that the differences between the two are related to the maturity and the breadth of the federal network and the similarity of the actors of the state network, even though both are recent phenomena. As a proposal for future research, a longitudinal study is suggested that accompanies the networks in their institutionalization process.

Keywords: innovation in the public sector, governance networks, innovation networks 


\section{Introdução}

As redes de governança são vistas como estratégias para organização e fomento da inovação no setor público brasileiro. A inovação é, atualmente, um tema recorrente nas organizações privadas e públicas a nível mundial, como instrumento estratégico na busca dos resultados (FARIA et al., 2017). No setor público, ela vem sendo percebida como uma área de interesse capaz de contribuir para o crescimento econômico e para a elevação da capacidade governamental de resolver problemas por meio do incremento da qualidade e da eficiência do serviço público (CAVALCANTE; CAMÕES, 2017; ISIDRO, 2018; MONTEZANO; ISIDRO, 2020).

As questões a serem abordadas, entretanto, são complexas e perpassam inúmeras dimensões, podendo ser entendidas como o que a literatura define de problemas perversos (wicked problems). A governança é um mecanismo frequentemente utilizado em situações envolvendo tal tipo de questões, nas quais há vários atores envolvidos, as informações são limitadas e fragmentadas e não há consenso sobre a natureza do problema e a solução desejada (KLIJN, 2012).

Embora haja consenso acerca da importância das redes nesses cenários, no âmbito da inovação, essas ainda são recentes no Brasil e no setor público. Conforme argumentam Faria et al. (2017, p. 242), "percebe-se a falta de preparo do governo para inovar sistematicamente", uma vez que seria necessário, nos termos de Isidro (2018), a consolidação de um ambiente de inovação, estimular as capacidades de inovação, bem como monitorar e avaliar as atividades e resultados da inovação na seara pública.

Assim, faz-se necessário debruçar sobre a questão e compreender melhor a articulação entre os atores e os processos que perpassam a rede. Nesse contexto, o artigo busca compreender a formação de redes de governança voltadas para inovação governamental no Brasil. A partir de duas dimensões de estudo - atores e processo -, as variáveis qualitativas analisadas para a dimensão atores foram participantes responsáveis e nível de engajamento. E como variáveis qualitativas da dimensão processo foram considerados o tempo de existência das redes, o tipo de interação e o processo decisório.

O presente artigo utiliza perspectiva descritiva do fenômeno estudado, partindo de uma metodologia que abarca revisão bibliográfica da temática da inovação e das redes de governança. Além disso, a fim de compreender melhor as redes de inovação 
governamental selecionadas, realizou-se uma análise documental baseada em dados empíricos e secundários associados aos dois casos estudados.

\section{Aspectos Teóricos}

\subsection{Redes de Inovação Governamental}

$\mathrm{O}$ conhecimento e as redes interorganizacionais são elementos que se complementam, potencializando a geração de valor. Cunha e Passador (2007) estabelecem que o compartilhamento do conhecimento é uma forma de dada entidade obter o conhecimento de forma mais rápida e eficiente, tendo em vista que as capacidades para a geração do conhecimento não dependem apenas das capacidades internas da organização. Tal argumento também é sugerido por Pereira (2005), segundo o qual os relacionamentos de parceria ou cooperação buscam melhorar a capacidade das organizações nas suas relações de interdependências, na tentativa de melhorar a eficácia organizacional.

Alguns autores colocam que as fontes de inovação podem ser encontradas nas relações externas da organização, ou seja, por meio de outras empresas (POWELL, 1987). Nessa linha, encorajam-se as entidades a se unirem na formação dessas redes de contato, as quais podem trazer benefícios diversos aos envolvidos, por meio da obtenção do conhecimento compartilhado (FLEURY; FLEURY, 2005).

Entretanto, a inovação não está ligada apenas a produtos e processos, mas também, e essencialmente, à compreensão e obtenção de conhecimento sobre a forma como as entidades se organizam e interagem nesse ambiente. Ou seja, não basta inovar em produtos ou em processos produtivos, há a necessidade de se compreender o papel da organização nesse contexto e inovar em termos de comportamento organizacional frente às mudanças e exigências ambientais (CUNHA; PASSADOR, 2007; ISIDRO, 2018).

Destaca-se ainda uma outra mudança proveniente dessa realidade, que traz consigo a formação de um ambiente social em que a confiança emerge como elemento propulsor e de fundamental importância para a transmissão do conhecimento entre as organizações, principalmente quando o conhecimento é o ativo de valor central. Assim, a confiança também é necessária para se trabalhar com o conhecimento numa relação interorganizacional (PoLITIS, 2003). 
A inovação e a busca pela intensificação do fluxo de informações mostram-se cada vez mais presentes no setor público. Especificamente no Brasil, as redes voltadas para a inovação no setor público, objeto de estudo neste trabalho, constituem elas mesmas um processo inovador (FARIA et al., 2017), já que iniciativas dessa natureza que envolvam a participação espontânea de servidores e instituições públicas são incipientes no Brasil (MONTEZANO; ISIDRO, 2020).

Cavalcante e Camões (2017, p. 126) apresentam um levantamento no qual "a inovação é um resultado da interação entre diferentes níveis (individual, organizacional, setor público como um todo e a sociedade) e fatores (pessoas, conhecimento, formas de trabalho e regras e processos), embora as fronteiras não sejam normalmente precisas".

Diante dessa perspectiva, ressalta-se a multiplicidade de atores envolvidos e de elementos que se delineiam na definição da inovação e de seu desenvolvimento. A partir dessa conceituação, a adoção de mecanismos de governança, como as redes interorganizacionais nos sistemas de inovação, merece destaque.

Para Faria et al. (2017), existe uma contraposição entre a cultura do serviço público que naturalmente é organizada de forma hierarquizada e a condução de projetos voltados para inovação em uma rede cuja estrutura é autônoma e transversal. Portanto, faz-se necessária a formação de uma 'cultura da inovação' no setor público para reconfigurar a hierarquização instituída pelo modelo weberiano burocrático e partir para novos formatos de gestão, como as redes de governança.

\subsection{Redes de Governança}

As redes de governança referem-se ao fenômeno empírico em que questões políticas são solucionadas por uma rede de atores, enquanto a governança em si está vinculada ao processo de interação e de orientação que ocorre nessas redes de atores (KLIJN, 2010). Dessa forma, a governança está associada à atuação em redes por envolver "formas de direção mais ou menos horizontais que ocorrem em redes de atores essencialmente interdependentes" (KLIJN; VAN BUUREN; EDELENBOS, 2012, p. 295).

Sørensen e Torfing (2007) caracterizam as redes como a articulação entre atores públicos e privados interdependentes, mas operacionalmente autônomos, os quais interagem em ambientes normativo e cognitivamente regulados, facilitando a 
autorregulação em oposição à hierarquia. Em trabalho mais recente, Klijn e Koppenjan (2016) apresentam a seguinte definição:

Redes de governança como padrões mais ou menos estáveis de relações sociais entre atores mutuamente dependentes, que se agrupam em torno de problemas de políticas, um programa de políticas e / ou um conjunto de recursos e que são formados, mantidos e alterados através de uma ou mais séries de interações (KLIJN; KOPPENJAN, 2016, p. 11, tradução nossa) $)^{1}$.

Na resenha de Gerrits e Verweij (2017) sobre o trabalho de Klijn e Koppenjan (2016) - Governance networks in public sector -, os autores alegam como argumento favorável para a formação de redes de governança o fato de que diferentes tipos de atores precisam operar em redes em função da necessidade de trocar recursos para alcançar objetivos compartilhados. Para Klijn e Koppenjan (2016), a emergência de redes de governança resulta da incapacidade de governos, empresas e sociedade civil de solucionarem os problemas sozinhos.

Pela perspectiva das redes de inovação governamental, o incremento na organização, nos processos e nos serviços tem reflexos diretos na população e nos mais diversos segmentos da sociedade. Diante dessa diversidade de envolvidos, a participação de vários atores da esfera pública e privada é fundamental, pois permite ampliar a troca de informações e a maximização dos recursos disponíveis. A resolução de questões por meio de redes governamentais requer o engajamento de diversas partes para composição da rede de governança. Contudo, como demonstrado por Fung (2006), o processo de seleção impacta a definição dos atores envolvidos na governança, sendo importantes, também, os canais de comunicação e os mecanismos para tomada de decisão. Essas dimensões são fundamentais para compreender não só quem participa, mas também para definir o grau de adesão e de comprometimento.

Além da importância da definição dos atores, Klijn, Van Buuren e Edelenbos (2012) destacam que as redes de governança envolvem atores com diferentes formas de processo decisório e construção do conhecimento, sendo necessária a convergência entre o nível de percepções, valores e cognições para obtenção de consenso. Nesse sentido, as redes são vistas como um meio para organização de procedimentos de aprendizado ou reflexão. Os autores ressaltam alguns comportamentos dos atores que precisam ser

\footnotetext{
${ }^{1}[\ldots]$ governance networks as more or less stable patterns of social relations between mutually dependent actors, which cluster around a policy problem, a policy programme, and/or a set of resources and which emerge, are sustained, and are changed through a series of interactions.
} 
suscitados na análise proposta, a saber: a tendência à prevalência de atitudes pragmáticas em razão da dificuldade de estabelecer o consenso genuíno; a escolha errática e as estratégias imprevisíveis dos atores; a possibilidade de seleção restritiva dentro da própria rede, por meio da ativação dos diferentes atores nos processos decisórios; e a restrição do impacto reflexivo aos representantes da organização que atuam na rede (KLIJN; VAN BuUREN; EdELENBos, 2012). Este último ponto deve ser enfatizado, uma vez que pode significar limitação do impacto das ações da rede, apesar da integração formal da organização.

Outro aspecto importante no relacionamento entre os participantes para o desenvolvimento exitoso da rede, segundo Klijn (2010), é a confiança, definida como percepção positiva dos atores sobre as intenções de outros atores, ou a percepção de que outros atores não terão comportamentos oportunistas (KLIJN, 2010). A confiança permite reduzir os custos de transação; facilita a cooperação e a estabilidade nas relações (de rede); estimula a aprendizagem e troca de conhecimento e favorece a inovação. Assim, é indispensável a consideração desse aspecto na análise proposta.

Do ponto de vista dos processos, McGuire e Agranoff (2011) destacam que os custos envolvidos nas interações são barreiras que requerem definição de agendas realistas, respeito às expectativas e foco nos resultados através da participação. Além disso, no que tange às redes públicas, os autores destacam que há impossibilidade de escapar de questões relacionadas a quão boas são as redes em uma perspectiva da performance e da efetividade dos programas e das ações desenvolvidas.

O gerenciamento da rede engloba quatro categorias centrais: organização (estratégias para coordenar as interações nas redes de governança em estruturas organizacionais temporárias); exploração de conteúdo (considerar as diferentes visões de diferentes atores, as novas soluções que podem surgir das interações com eles, conectando as ideias de diferentes atores); conexão (assegurar contatos entre atores, melhorando relações); e acordos de processo (definir regras de processo e métodos de interação entre os atores) (KLIJN, 2010). Sem perpassar tais conjuntos, a complexidade das interações escapa à gestão da rede, ocasionando o risco de atuação ineficaz para a atividade de administração.

O’Toole e Meier (2010) evidenciaram que o nível de interação e a qualidade do gerenciamento impactam a rede através do grau e do tipo de interações desenvolvidas, sendo crucial a qualidade do gerente e a capacidade deste de adequar-se ao ambiente, à 
dimensão política e aos cenários de crise. Assim, é indispensável considerar também quem são os responsáveis pelo processo e pelas interações.

Os aspectos metodológicos da pesquisa serão apresentados na próxima seção.

\section{Aspectos Metodológicos}

Este é um estudo de natureza qualitativa, atendendo ao caráter descritivo por expor as características de um fenômeno em específico (COLLIS; HuSSEY, 2005): redes de governança para a inovação no setor público.

Quanto aos meios, pretende-se realizar um estudo de dois casos, analisando redes de governança para inovação no setor público - a Rede InovaGov do Governo Federal e a Comunidade de Simplificação do Governo de Minas Gerais. A escolha pelo estudo de caso ocorre ao examinar acontecimentos contemporâneos, porém os comportamentos relevantes não podem ser manipulados (YIN, 2001), tal como ocorre com as redes de inovação. Para compreender a formação de redes de governança voltadas para inovação no setor público, foram estipulados como objetivos específicos: (1) explicar o surgimento de redes de inovações governamentais pela revisão de literatura sobre o tema; (2) descrever cada um dos casos em análise - Rede InovaGov e a Comunidade de Simplificação; (3) realizar uma análise comparativa entre os casos por meio de dimensões de análise; e (4) identificar similitudes e diferenças entre as redes estudadas. O percurso metodológico seguido por esta pesquisa está ilustrado na Figura 1:

\section{Figura 1- Percurso Metodológico da Pesquisa}

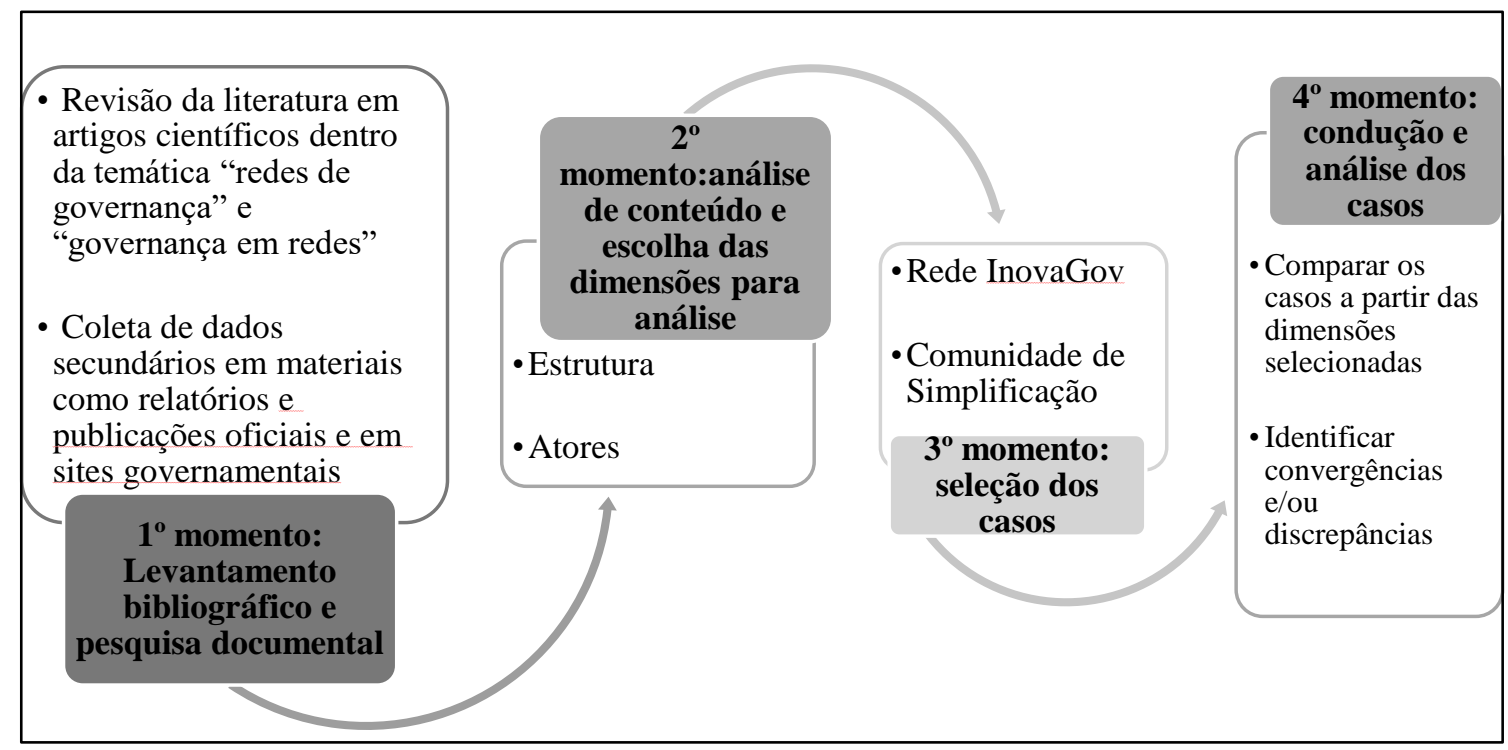

Fonte: Elaboração própria. 
A descrição desse percurso metodológico pode funcionar enquanto um protocolo. Como protocolo do estudo dos casos selecionados - a Rede InovaGov e a Comunidade de Simplificação -, duas dimensões de análise foram escolhidas para estruturar este estudo, de maneira que seja possível reaplicá-lo em outros casos envolvendo redes de governança orientadas para inovações governamentais. Segundo Martins (2008), a construção de um protocolo precede a análise e possibilita uma aproximação com os casos, bem como a descrição das ações desenvolvidas até o fim do estudo.

Dessa forma, a pesquisa inicia-se com o levantamento bibliográfico acerca das redes de inovação e da governança em rede, tema sobre os quais foram analisados os principais trabalhos e os mais recentes (FUNG, 2006; KLIJN, 2010; OsBORNE, 2010; MCGuire; AGranofF, 2011; TORFInG, 2012; KliJn; VAN BuUREN; Edelenbos, 2012; KLIJN; KoPPENJAN, 2016; IsIDRO, 2018; MonTEZANo ; IsIDRO, 2020). Paralelamente, os dados secundários deste estudo foram coletados em: (a) materiais institucionais governamentais, como os relatórios produzidos pelo Instituto de Pesquisa Econômica Aplicada (Ipea) sobre inovação no setor público e publicações elaboradas pelo governo mineiro sobre as ações pertinentes à realização de inovações para a administração pública no estado (FARIA et al. 2017; Minas Gerais, 2018; CAVAlCANTE; MendonÇA; BRANDALISE, 2019); (b) sítios eletrônicos oficiais das duas redes; e (c) regulamentos que respaldam a oficialização das redes. Quanto a este último, somente o documento sobre a Rede InovaGov (Conselho DA JustiçA FEDERAL, 2018) foi localizado no período da pesquisa. Dado o ineditismo do tema, as informações obtidas ainda são preliminares.

Conforme exposto pela Figura 1, no primeiro momento da pesquisa houve a exploração do material coletado. Na sequência, realizou-se a análise de conteúdo dessas informações (BARDIN, 1977) para identificar elementos relevantes na teoria sobre as redes de governança que fossem aplicáveis à realidade das redes de inovação no setor público.

Com a análise de conteúdo, a pesquisa avança para um segundo momento que foi a definição das categorias de análise. Portanto, as duas dimensões derivam da revisão bibliográfica acerca das redes de governança no setor público e da coleta de dados preliminar dos casos. A primeira dimensão volta-se para os atores que participam das redes indicadas, destacando-se alguns pontos centrais: quantos são os atores, quem está na rede, quais os interesses envolvidos e qual o nível de engajamento dos participantes. Esta última categoria consolidou os graus de adesão, comprometimento com as ações compartilhadas e a confiança nas interações, os quais foram classificados individualmente 
como alto, médio ou baixo e combinados para avaliação referente aos casos. Aqui, o engajamento é analisado quando da entrada dos atores na rede, ou seja, inicialmente, um possível indicativo da motivação dos atores para aderirem a tais redes.

A segunda dimensão é a de processos, perpassando o gerenciamento das redes; o tempo de existência destas; os objetivos comuns; o tipo de interação entendido como a forma de compartilhamento de experiências, ideias, conhecimento ou atuação conjunta, entre outros; e as formas de delegação para a tomada de decisão (horizontalizada e participativa ou verticalizada e hierarquizada).

No terceiro momento, os casos selecionados foram Rede InovaGov e Comunidade de Simplificação. A justificativa para essa seleção é a relevância da constituição dessas redes para inovações no contexto da administração pública brasileira. Embora sejam dois casos, o que dificulta o potencial de generalização, esses casos são exemplares e ilustram bem como as redes de governança são formadas, principalmente para a gestão pública nas esferas federal e estadual. Também se faz necessário ressaltar que são fenômenos recentes e, por isso, novos casos de redes de inovação para o setor público ainda estão emergindo. O último momento deste estudo consistiu na análise dos dados coletados de maneira comparativa, indicando semelhanças e diferenças. Os resultados são expostos na seção seguinte.

\section{Apresentação e Análise de Resultados}

Os estudos da Rede InovaGov e da Comunidade de Simplificação são analisados por meio de duas dimensões - atores e processos. O esquema apresentado no Quadro 1 sintetiza os principais elementos apurados no escopo da pesquisa. 
Quadro 1- Comparativo entre redes de governança para inovação no setor público - Rede InovaGov e Comunidade de Simplificação.

\begin{tabular}{|c|c|c|c|}
\hline \multirow{2}{*}{$\begin{array}{l}\text { Dimensões de } \\
\text { análise }\end{array}$} & \multirow{2}{*}{ Variáveis } & \multicolumn{2}{|c|}{ Redes de inovação } \\
\hline & & Rede InovaGov & Comunidade de Simplificação \\
\hline \multirow{4}{*}{ ATORES } & Tamanho & $\begin{array}{l}111 \text { organizações atualmente, inclusive Seplag-MG. Em } \\
2016,187 \text { pessoas. }\end{array}$ & 985 membros em 2018. \\
\hline & Tipos & $\begin{array}{l}\text { Instituições públicas, e suas vinculadas, de todos os poderes } \\
\text { e entes da Federação; instituições do setor privado; } \\
\text { instituições de ensino e pesquisa; organizações do terceiro } \\
\text { setor; e pesquisadores independentes. }\end{array}$ & Servidores públicos estaduais \\
\hline & Órgão responsável & $\begin{array}{l}\text { Ministério do Planejamento, Desenvolvimento e Gestão } \\
\text { (MP) - Departamento de Modernização em Gestão Pública } \\
\text { (Inova) }\end{array}$ & $\begin{array}{l}\text { Secretaria de Planejamento e Gestão (Seplag) do Estado de } \\
\text { Minas Gerais - Superintendência Central de Inovação e } \\
\text { Modernização Institucional (SCIM) }\end{array}$ \\
\hline & $\begin{array}{l}\text { Nível de engajamento } \\
\text { inicial dos atores }\end{array}$ & Alto & Médio-Baixo \\
\hline \multirow{6}{*}{ PROCESSO } & Ano de criação & $2015-2016$ & 2017 \\
\hline & Tempo de existência* & Aproximadamente 3 anos & 1 ano e 3 meses \\
\hline & Objetivos & Melhorias na prestação de serviços públicos & $\begin{array}{l}\text { Desenvolver iniciativas voltadas para a desburocratização, } \\
\text { modernização e inovação na gestão pública do Estado }\end{array}$ \\
\hline & Modalidade de interação & $\begin{array}{l}\text { Compartilhamento de ideias, experiências, conhecimento } \\
\text { Capacitação e treinamento } \\
\text { Atuação conjunta }\end{array}$ & $\begin{array}{l}\text { Compartilhamento de ideias, experiências, conhecimento } \\
\text { Capacitação e treinamento }\end{array}$ \\
\hline & \multirow{2}{*}{$\begin{array}{l}\text { Delegação da tomada de } \\
\text { decisão }\end{array}$} & Descentralizado & Centralizado \\
\hline & & Autonomia e horizontalidade & Verticalidade \\
\hline
\end{tabular}

Fonte: Elaboração própria com base na revisão da literatura e nos dados da pesquisa. * Considerando o período de coleta de dados - nov/2018 a fev/2019. 


\subsection{Rede InovaGov}

A Rede InovaGov foi constituída por iniciativa do Ministério do Planejamento, Desenvolvimento e Gestão (MP) do Governo Federal brasileiro em parceria com o Tribunal de Contas da União (TCU) e do Conselho da Justiça Federal (CJF), por meio de um Acordo de Cooperação Técnica (ACT), celebrado em 11 de outubro de 2016. Todavia, a gênesis da rede está relacionada às primeiras discussões organizadas pelo MP, principalmente com o evento da I Semana de Inovação em Gestão Pública realizada ao final de 2015 (FARIA et al., 2017). Logo, a InovaGov tinha pouco mais de três anos de existência na época da pesquisa.

A rede é composta por: atores governamentais de diversas entidades dos três Poderes e também de outros entes federativos (estadual e municipal), atores da iniciativa privada, atores do terceiro setor e membros da comunidade acadêmica. Nessa rede, os membros se propõem a contribuir com a construção colaborativa de soluções para a inovação no setor público (CONSELHO DA JUSTIÇA FEDERAL, 2018).

Pela Tabela 1, é possível mensurar o quantitativo de organizações e instituições componentes da rede.

\section{Tabela 1- Quantidade de organizações membros da rede InovaGov}

\begin{tabular}{lc}
\hline \multicolumn{1}{c}{ Atores } & Quantidade (n' de organizações) \\
\hline Setor público & 76 \\
Setor privado & 20 \\
Terceiro setor & 13 \\
Academia & 5 \\
Total & 114 \\
\hline
\end{tabular}

Fonte: Brasil, MP, Porta InovaGov, ago-2020.

Além disso, há que se considerar os participantes individuais dentro de cada órgão e os pesquisadores independentes do setor acadêmico, cuja quantidade não foi divulgada pelos canais oficiais eletrônicos da Rede InovaGov. Pelos dados de Faria et al. (2017), em outubro de 2016, a rede contava com 38 instituições públicas e 187 pessoas. Nota-se a diversidade de atores nessa rede, cujo principal objetivo é contribuir para melhorias na prestação de serviços públicos. 
A partir do InovaGov, projetos conjuntos são realizados para disseminar a cultura de inovação, seja na aquisição de inovações no setor público ou na verificação de práticas inovadoras e bem-sucedidas no setor privado (PORTAL INOvaGov, 2020). Um dos projetos em andamento desde 2018 é a realização de um estudo para o Sistema de Inovação do Serviço Público do Brasil em parceria com a Organização para a Cooperação e Desenvolvimento Econômico (OCDE) (PorTAl InOvaGov, 2020).

Quanto aos processos, a Rede InovaGov é norteada por “desafios mobilizadores". Esses desafios foram constituídos para ressaltar o caráter horizontal da rede devido à demanda por uma atuação transversal de diversos órgãos. Os desafios mobilizadores foram posteriormente delimitados em objetivos específicos e se constituíram em projetos (FARIA et al., 2017).

Um dos desafios foi a articulação de iniciativas e programas de inovação. Uma das medidas foi a reformulação do Prêmio de Inovação em Gestão Pública, organizado pela Escola Nacional de Administração Pública (Enap), bem como a promoção de maior adesão de possíveis candidatos ao projeto Poder de Compra do Estado na Aquisição de Inovação - Propostas de Melhoria para Incorporação de Inovação, projeto que envolveu a participação do Ministério do Desenvolvimento, Indústria e Comércio (MDIC), inclusive para fomentar parcerias internacionais (FARIA et al., 2017). Logo, esses desafios possibilitaram maior adesão e engajamento de novos atores, além de delegar a responsabilidade que era inteiramente do Departamento de Modernização em Gestão Pública (Inova) do Ministério do Planejamento aos demais atores em projetos específicos.

\subsection{Comunidade de Simplificação}

A Comunidade de Simplificação é uma rede criada e coordenada pela Superintendência Central de Inovação e Modernização Institucional (SCIM) da Secretaria de Planejamento e Gestão (Seplag) do Estado de Minas Gerais, que objetiva a desburocratização, a modernização e a inovação na gestão pública mineira (MINAS GERAIS, 2018). A Comunidade de Simplificação foi lançada em 29 de agosto de 2017 (PORTAl SePlag, 2018), tendo um ano e três meses de existência na época da pesquisa.

Segundo o Guia para Inovação na Gestão Pública - Coletânea de Inovação e Modernização na Gestão Pública, Volume 1 (MinAs GERAIS, 2018), elaborado por servidores da SCIM com a colaboração de especialistas em inovação, a Comunidade de 
Simplificação é composta por um conjunto de servidores estaduais cuja interação ocorre em espaços presenciais e virtuais. Até o período de realização desta pesquisa, a Comunidade de Simplificação possuía 985 membros, todos pertencentes a instituições do Governo de Minas (PorTal SEPLAG, 2018).

A interação entre os atores resulta em discussões, troca de experiências, divulgação de eventos e promoção de treinamentos e capacitações, como os cursos de melhoria de processos e Design Thinking ${ }^{2}$. Além da rede, a SCIM também é responsável pelo Prêmio Inova Minas Gerais, que seleciona e premia as ideias e iniciativas inovadoras concebidas por servidores públicos estaduais (MINAS GERAIS, 2018).

\subsection{Análise dos Atores nas Redes de Inovação}

Em ambas as redes, os atores governamentais se fazem presentes, tal como pode ser visto no Quadro 1. Todavia, a Rede InovaGov possui uma gama de atores mais diversificada, uma vez que engloba agentes públicos de todos os poderes (Executivo, Legislativo e Judiciário) e de todas as instâncias (União, estados e municípios). Inclusive a própria Seplag-MG, órgão responsável pela criação da Comunidade de Simplificação, é membro da InovaGov.

Além do mais, a InovaGov também abrange outros atores dos demais segmentos, como da iniciativa privada, do terceiro setor e da comunidade acadêmica, em consonância com a própria definição de redes de governança (SøRENSEN; TORFING, 2007; TORFING, 2012; KLIJN; KopPEnJan, 2016), que inclui a diversidade de atores. Já a Comunidade de Simplificação se restringe a servidores públicos estaduais, o que limita sua atuação. Em termos quantitativos, a Comunidade de Simplificação tem uma quantidade maior de atores individuais, 985 membros, porém a InovaGov apresenta mais heterogeneidade de atores e um número maior de instituições envolvidas, 111 no total em 2020.

Quanto à responsabilidade perante as redes de inovação, nos dois casos esse papel ficou atribuído aos órgãos de planejamento: o Ministério do Planejamento,

\footnotetext{
${ }^{2}$ Segundo Cavalcante, Mendonça e Brandalise (2019) em seu artigo sobre a aproximação entre o Design Thinking e a elaboração e a implementação de políticas públicas, o Design Thinking é uma abordagem que denota a compreensão dos problemas de forma aprofundada bem como a construção de possíveis soluções para esses problemas. O Design Thinking possui três princípios: a) foco no ser humano, na inclusão de todos os colaboradores no processo (o político, o gestor até o cidadão na outra ponta); b) a cocriação de soluções que geram possibilidades aos problemas enfrentados; e c) a experimentação quando as ideias são testadas e que a prototipagem é uma fonte de aprendizado - realiza-se o protótipo antes de implementar determinada solução (CAVALCANTE, MENDONÇA, BRANDALISE, 2019).
} 
Desenvolvimento e Gestão (MP) do Governo Federal, no caso da Rede InovaGov; e a Secretaria de Planejamento e Gestão (Seplag) do Estado de Minas Gerais, na Comunidade de Simplificação. Isso se justifica pelo fato de os órgãos de planejamento serem responsáveis pela formulação de políticas públicas de inovação no setor público. Em ambos os órgãos há setores para tal finalidade - Departamento de Modernização em Gestão Pública (Inova) do MP e a Superintendência Central de Inovação e Modernização Institucional (SCIM) da Seplag-MG. Contudo, há uma variedade de atores atuantes na InovaGov que assumiram a responsabilidade por projetos específicos, patrocinando-os, vide o caso da premiação da Enap e do MDIC para o projeto de Compras para Aquisição de Inovações (FARIA et al., 2017). Isso possibilitou minimizar o protagonismo do MP na rede, embora ainda seja o órgão responsável. Já na Comunidade de Simplificação, a Seplag está integralmente responsável pelos projetos desenvolvidos, inclusive tendo que insistir que os atores participantes atuem de forma mais incisiva.

Nota-se aqui a relevância da observação de Fung (2006), que ressalta a importância da definição dos atores envolvidos na governança, compreendendo quem participa e qual o grau de adesão dos atores. No contexto da inovação do setor público, a oposição apontada por Faria et al. (2017) entre a estrutura hierárquica do setor público culturalmente instituída e a condução de redes de forma transversal e autônoma também se verifica em ambos os casos.

\subsection{Análise do Processo do Gerenciamento das Redes de Inovação}

O processo de gerenciamento das redes de inovação perpassa desde a formação da rede até a gestão e a forma de interação dos atores inseridos nas redes. Com relação à criação e ao tempo de existência, o Quadro 1 demonstra que a Rede InovaGov é mais antiga, sendo concebida no final de 2015 e atuante desde então. Já a Comunidade de Simplificação é mais recente, fundada em agosto de 2017. O tempo de existência na rede impacta no desenvolvimento de maior engajamento e, posteriormente, de maior confiança.

A construção de confiança dentro das redes de governança requer instrumentos que assegurem que os atores participantes se abstenham de comportamentos oportunistas (KLIJN, 2010). Para minimizar essa questão, principalmente no caso do setor público, cuja “cultura de desconfiança em relação ao servidor" permeia as relações (FARIA et al. 2017, 
p. 246), atrelada à presença de atores externos ao serviço público na rede, o Ministério do Planejamento firmou um Acordo de Cooperação Técnica com o TCU e o CJF em outubro de 2016 com o objetivo de formalizar a rede. Essa formalização foi impactada pela cultura organizacional e pelo sentimento de instabilidade (FARIA et al., 2017) proporcionado por características da atuação em redes como a fluidez e a horizontalidade em detrimento da hierarquia comumente presente nas organizações públicas. Isso está adequado ao Referencial Básico de Governança (BRASIL-TCU, 2014), em que as redes transorganizacionais estão inseridas na vertente político-administrativa de governança no setor público. E, segundo Stoker (1998), tais redes ultrapassam as barreiras funcionais das organizações. Nessa acepção, a capacidade dos governos na formulação e na implementação de políticas públicas é aprimorada ao estabelecer relações e parcerias com organizações públicas e privadas (BRASIL-TCU, 2014, p.20).

As quatro categorias de gerenciamento de redes mencionadas por Klijn (2010) organização, exploração de conteúdo, conexão e acordos de processo - são visíveis na formalização do InovaGov, observando-se a convergência apontada por Klijn, Van Buuren e Edelenbos (2012) entre o nível de percepções, valores e cognições para obter consenso entre os atores.

Quanto à modalidade de interação, ambas as redes demonstraram ações voltadas para o compartilhamento de ideias, experiências, conhecimento e para a realização de capacitação e treinamento, sendo que membros da Rede InovaGov capacitaram membros da Comunidade de Simplificação em metodologias e ferramentas, como o Design Thinking. Vislumbra-se a importância do compartilhamento do conhecimento (FLEURY; FLEURY, 2005) entre redes, uma vez que a capacidade do conhecimento não é obtida apenas de forma interna dentro das organizações (CUNHA; PASSADOR, 2007), mas sim pela troca.

A interação entre os atores da InovaGov ocorre de forma mais autônoma e automática, enquanto na Comunidade de Simplificação, a SCIM ainda exerce um controle forte sobre os atores envolvidos na rede. Tal comportamento das redes repercute na forma como a decisão acontece. Na Rede InovaGov, foi constatado um declínio da centralidade do MP, e os atores que patrocinaram determinados projetos "assumiram as rédeas" de algumas ações. Já na Comunidade de Simplificação, a SCIM ainda funciona conforme uma hierarquia, com a Seplag no comando das ações, o que vai contra a formatação de redes, que precisam da transversalidade e da horizontalidade das relações. A delegação 
da decisão por parte do MP para os participantes da rede permite uma atuação mais conjunta, mesmo que seja pontual. É o começo para uma nova forma de interação em redes de governança voltadas para inovação no serviço público brasileiro.

\section{Considerações Finais}

Analisando a formação de redes de governança no setor público brasileiro como estratégia para o estímulo e o desenvolvimento de inovação, o presente trabalho buscou identificar e analisar, a partir das referências da literatura, as questões complexas e as dimensões que permeiam as duas iniciativas. Foram selecionadas para o estudo uma rede estruturada no nível federal - a Rede de Inovação do Setor Público (InovaGov) - e outra no nível estadual em Minas Gerais - a Comunidade de Simplificação.

O referencial teórico engloba tanto conceitos diretamente ligados à inovação, quanto as teorias e referências bibliográficas de redes de governança. Em relação aos dados, esses foram obtidos através de pesquisas nos sítios eletrônicos oficiais de ambas as redes, nos relatórios produzidos pelos órgãos responsáveis, nas legislações e nos regulamentos específicos da formação das redes. A limitação de conteúdo sobre as redes InovaGov e Comunidade de Simplificação justifica-se pelo fato de serem ambas um fenômeno recente, o que agrega valor a este trabalho.

Duas dimensões nortearam o estudo proposto das redes de inovação: uma referente aos atores que participam das redes indicadas, destacando-se quem está nas redes, quais os interesses envolvidos, qual o grau de adesão e comprometimento dos participantes e o engajamento; e uma dimensão relativa aos processos, que verifica o gerenciamento das redes, o tempo de existência e o tipo de interação ao considerar compartilhamento de experiências, ideias, conhecimento ou atuação conjunta.

Observou-se que há distinções significativas nas redes no que tange à forma de construção, de gerenciamento e aos tipos de atores presentes em cada uma, gerando implicações diretas na capacidade de atuação e impacto das estruturas. Outro ponto que merece destaque é o nível de engajamento dos atores, o qual tem reflexos na própria autonomia, materializada na delegação da tomada de decisão em cada uma das redes, tal como foi constatado pela atuação mais autônoma e horizontalizada da Rede InovaGov em comparação com a Comunidade de Simplificação. Essas diferenças podem ser explicadas pela maturidade de cada uma das redes de inovação, entendida como o tempo 
de existência destas, combinado com a incorporação dos conceitos e efetivação dos elementos que definem as redes de governança.

Entretanto, para se verificar a confiabilidade dos atores inseridos nas redes, seria necessário um tempo maior para averiguar a institucionalização da rede, o que envolve o comportamento dos participantes, bem como a capacidade de adesão de novos membros e a continuidade de seus objetivos. Portanto, um estudo longitudinal para analisar a atuação dos atores dentro de redes de governança para a inovação do setor público, e não somente a formação das mesmas, fica como sugestão para futuras pesquisas.

\section{Referências}

BARDIN, Laurence. Análise de conteúdo. Lisboa: Edições 70, 1977.

BRASIL. Tribunal de Contas da União. Governança pública: referencial básico de governança aplicável a órgãos e entidades da administração pública e ações indutoras de melhoria. Brasília: TCU, Secretaria de Planejamento, Governança e Gestão, 2014. 80 p.

Cavalcante, Pedro; Camões, Marizaura R. S. Public innovation in Brazil: an overview of its types, results and drivers. Brasília: Ipea, 2017. p. $1-36$.

CAvalcante, Pedro; MendonçA, Letícia; BRANDALISE, Isabella. Políticas públicas e design thinking: interações para enfrentar desafios contemporâneos. In: CAVALCANTE, Pedro (Org.). Inovação e políticas públicas: superando o mito da ideia. Brasília: Ipea, 2019. p. 29-52.

CONSElHo DA JustiçA Federal (CJF). Regulamento da Rede Federal de Inovação no Setor Público - InovaGov. 2018. Disponível em: $<$ http://inova.gov.br/wpcontent/uploads/2018/10/Regulamento-RedeInovaGov.pdf >. Acesso em: 25 nov. 2018.

COllis, Jill; Hussey, Roger. Pesquisa em Administração. Porto Alegre: Bookman, 2005.

CunHA, Júlio Araújo Carneiro da; PASSADOR, João Luiz. Alianças estratégicas de organizações: o aprendizado institucional na formação de redes. Revista Eletrônica de Ciência Administrativa, v. 6, n. 1, p. 1-16, 2007.
EdELENBos, Jurian; KLIJN, Erik-Hans. Trust in complex decision-making networks: a theoretical and empirical exploration. Administration \& Society, v. 39, n. 1, p. 25-50, 2007.

FARIA, Yana de; ITABORAHY, Felipe Bragança; PALVARINI, Bruno Carvalho; ENDO, Iara Cremonesi; RoncaratTI, Luanna Sant'Anna. Experiências da rede de inovação no setor público (InovaGov). In: CAVAlCANTE, Pedro; CAMÕES, Marizaura; CUNHA, Bruno; SEVERo, Wilber (Orgs.). Inovação no setor público: teoria, tendências e casos no Brasil. Brasília: Ipea, 2017. p. 241-248.

Fleury, Afonso; Fleury, Maria Tereza Leme. A arquitetura das redes empresariais como função do domínio de conhecimentos. In: AмAтO Neto, João. (Org.). Redes entre organizações: domínio do conhecimento e da eficácia operacional. São Paulo: Atlas, 2005.

FUNG, Archon. Varieties of participation in complex governance. Public Administration Review, v. 66, p. 66-75, 2006.

GERRITS, Lasse; VERWEIJ, Stefan. When governance networks become the agenda. Public Administration Review, v. 77, n. 1, p. 144-146, 2017.

IsIDRO, Antonio. Gestão pública inovadora: um guia para a inovação no setor público. Curitiba: Editora CRV, 2018.

KLIJN, Erik Hans. Trust in governance networks: looking for conditions for innovative solutions and outcomes. In: OsBORNE, Stephen P. (Ed.). 
The new public governance? London: Routledge, 2010. p. 303- 321.

KLIJN, Erik Hans; VAN BUUREN, Arwin; EDELENBOS, Jurian. The impact of governance: a normative and empirical discussion. In: LEVIFAUR, David (Org.). The Oxford Handbook on Governance. Oxford: Sage, 2012.

KLIJN, Erik Hans. New Public Management and Governance: a comparison. In: LEVI-FAUR, David (org.).The Oxford Handbook on Governance. Oxford: Sage, 2012.

KLIJN, Erik Hans; KoPPENJAN, Joop. Governance networks in the public sector. London: Routledge, 2016.

MARTINS, Gilberto Andrade. Estudo de caso: uma reflexão sobre a aplicabilidade em pesquisas no Brasil. Revista de Contabilidade $e$ Organizações, v. 2, n. 2, p. 8-18, 2008.

MCGUIRE, Michael; AgRAnOFF, Robert. The limitations of public management networks. Public Administration, v. 89, n. 2, p. 265-284, 2011.

MinAs GerAIS. Secretaria de Planejamento e Gestão. Guia para inovação na gestão pública. Elaborado pela Superintendência Central de Inovação e Modernização Institucional. Belo Horizonte: Fundação João Pinheiro, 2018. 78p.

MonteZAno, Lana; IsIDRO, Antonio. Proposta de modelo multinível de competências para gestão pública inovadora. Future Studies Research Journal: Trends and Strategies, v. 12, n. 2, p. 355-378, 2020.

OSBORNE, Stephen P. The new public governance: a suitable case for treatment? In: OSBORNE, Stephen P. (Ed.). The new public governance? Emerging perspectives on the theory and practice of public governance. London: Routledge, 2010. p.1-17.

O'ToOLE, Laurence J.; MEIER, Kenneth J. Implementation and managerial networking in the new public governance. In: OSBORNE, Stephen P. (Ed.). The new public governance?
Emerging perspectives on the theory and practice of public governance. London: Routledge, 2010. p. 322-336.

PereirA, Breno Augusto Diniz. Estruturação de relacionamentos horizontais em rede. Porto Alegre, 2005. Tese (Doutorado em Administração) - UFRGS.

Portal DA REDE DE INOVAÇÃo NO SETOR PÚBLICO (InovaGov). Disponível em:< http://inova.gov.br/>. Acesso em: 25 mar. 2020.

Portal da Secretaria de Planejamento E GESTÃo (Seplag) - Comunidade de Simplificação. Disponível em: <http://www.planejamento.mg.gov.br/pagina/ge stao-governamental/inovacao/comunidade-desimplificacao>. Acesso em: 25 nov. 2018.

POWELL, Walter W. Hybrid organizational arrangements: new form or transitional development? California management review, v. 30, n. 1, p. 67-87, 1987.

PoLITIS, John D. The connection between trust and knowledge management: what are its implications for team performance. Journal of knowledge management, v. 7, n. 5, p. 55-66, 2003.

RHODES, Roderick Arthur William. The new governance: governing without government. Political studies, v. 44, n. 4, p. 652-667, 1996.

STOKER, Gerry. Governance as theory: five propositions. International social science journal, v. 50, n. 155, p. 17-28, 1998.

SøRENSEN, Eva; ToRFING, Jacob. Theoretical approaches to democratic network governance. In: SøREnSEN, Eva; TORFING, Jacob (Eds). Theories of democratic network governance. London: Palgrave Macmillan, 2007. p. 233-246.

TORFING, Jacob. Governance Networks. In: LEVI-FAUR, David (Org.). The Oxford Handbook on Governance. Oxford: Sage, 2012.

YIN, Robert. K. Estudo de caso: planejamento e método. Porto Alegre: Bookman, 2001. 


\section{Cristina Camila Teles}

\section{iD https://orcid.org/0000-0001-9848-1209}

Doutoranda em Administração pela UFMG. Mestra em Administração Pública pela FJP-MG. Especialista em Gestão de Pessoas pela UEMG. Bacharel em Gestão Pública pela UFMG e Bacharel em Relações Internacionais pela PUC-MG.

E-mail: cristina.c.t.saldanha@gmail.com

\section{Ana Luiza Santos Terra}

(iD https://orcid.org/0000-0002-5167-4289

Mestranda em Administração/Tecnologias Gerenciais na Universidade Federal de Minas Gerais (UFMG). Bacharel em Administração Pública pela Fundação João Pinheiro (FJP) e em Direito pela Universidade Federal de Minas Gerais (UFMG).

E-mail: terra.analuiza@gmail.com

\section{Marcus Vinicius Gonçalves da Cruz}

(iD https://orcid.org/0000-0003-2476-3822

Doutor e Mestre em Administração pela UFMG. Pós-Doutor no Instituto de Sociologia da Universidade do Porto. Doutorado Sanduíche na American University, Washington, D.C., Estados Unidos. Coordenador e Professor do Mestrado em Administração Pública da Escola de Governo da FJP-MG.

E-mail: marcus.cruz@fjp.mg.gov.br

\section{Carolina Gomes Rosado}

(iD https://orcid.org/0000-0003-1953-2907

Mestre em Administração pela UFV, Especialista em Direito Público pela PUC/MG. Bacharel em Direito pela UFV.

E-mail: carolinagomesrosado@gmail.com

\section{José Clemente Maria Ferreira}

iD https://orcid.org/0000-0002-9682-5378

Mestre em Controladoria e Contabilidade pela UFMG. Especialista em Gestão Fiscal e Administração Pública pela Faculdade Arnaldo. Bacharel em Ciências Contábeis pela Universidade Federal de Minas Gerais (UFMG).

E-mail: jose_clemente@outlook.com 